\title{
HIF-3 $\alpha$ affects preeclampsia development by regulating EVT growth via activation of the Flt-1/JAK/STAT signaling pathway in hypoxia
}

\author{
HONGMEI QU ${ }^{1 *}$, QUN YU ${ }^{1 *}$, BEI JIA ${ }^{1 *}$, WENZHE ZHOU ${ }^{1}$, YINGHONG ZHANG ${ }^{1}$ and LINSONG MU ${ }^{2}$ \\ Departments of ${ }^{1}$ Obstetrics and Gynecology and ${ }^{2}$ General Surgery and Pediatric Surgery, \\ The Affiliated Yantai Yuhuangding Hospital of Qingdao University, Yantai, Shandong 264000, P.R. China
}

Received November 27, 2019; Accepted May 27, 2020

DOI: $10.3892 / \mathrm{mmr} .2020 .11701$

\begin{abstract}
Preeclampsia (PE) is a common obstetric disease occurring after 20 weeks of gestation. Hypoxia-inducible factor (HIF)-3 $\alpha$ potentially functions as a regulatory factor in $\mathrm{PE}$ development, however its specific molecular mechanism remains to be elucidated. The present study aimed to investigate the function of HIF-3 $\alpha$ in trophoblast cell line HTR-8/SVneo, to provide a better understanding of the pathology and treatment of PE. Normal and PE placentas were obtained from pregnant women. HTR8/SVneo cells were cultured under the condition of normoxia or hypoxia, pretreated with or without AG490, then transfected with HIF-3 $\alpha$. The gene expression levels of HIF-3 $\alpha$ and Fms like tyrosine kinase receptor (Flt) 1 extracted from the placentas and cells were detected by reverse transcription-quantitative PCR, and the expression levels of proteins and Janus kinase signal transducer and activator of transcription (JAK/STAT) phosphorylation were detected by western blot analysis. Viability and apoptosis of the treated cells were assessed by MTT and flow cytometry. The results demonstrated that HIF-3 $\alpha$ and Flt-1 gene expression levels of PE placentas were reduced compared with normal placentas. Under a hypoxic environment, the expression
\end{abstract}

Correspondence to: Dr Yinghong Zhang, Department of Obstetrics and Gynecology, The Affiliated Yantai Yuhuangding Hospital of Qingdao University, 20 East Yuhuangding Road, Zhifu, Yantai, Shandong 264000, P.R. China

E-mail: yihzh_zh@163.com

Dr Linsong Mu, Department of General Surgery and Pediatric Surgery, The Affiliated Yantai Yuhuangding Hospital of Qingdao University, 20 East Yuhuangding Road, Zhifu, Yantai, Shandong 264000, P.R. China

E-mail: linson_mu@163.com

${ }^{*}$ Contributed equally

Key words: preeclampsia, extravillous cytotrophoblast, Fms-like tyrosine kinase receptor 1 , hypoxia-inducible factor- $3 \alpha$, Janus kinase-signal transducer 2/signal transducer and activator of transcription 3 signaling pathway levels of HIF-3 $\alpha$ and Flt-1, the phosphorylation of JAK/STAT and the cell viability of HTR8/SVneo cells were increased at first and then reduced, whereas cell apoptosis was promoted over time. Under chronic hypoxia, the expression levels of HIF-3 $\alpha$ and Flt-1, JAK/STAT pathway phosphorylation and cell viability of AG490-treated HTR8/SVneo cells were reduced, but cell apoptosis was promoted. However, the upregulation of HIF-3 $\alpha$ in HTR8/SVneo cells markedly reversed the effects of AG490 on the cells under hypoxia. Thus, the present study preliminarily demonstrated that HIF-3 $\alpha$ was involved in PE development by regulating extravillous cytotrophoblast growth via Flt-1 and the JAK/STAT signaling pathway.

\section{Introduction}

Preeclampsia (PE) is a heterogeneous disorder affecting $3-5 \%$ of all pregnancies (1-3). The disease is often diagnosed by the combination of hypertension and proteinuria in the second half of pregnancy (4-6). PE can cause maternal and perinatal death, and has high prevalence in developing countries $(4,7)$. Currently, delivering the placenta and neonate by induced labor is a widely used strategy for the treatment of PE patients, however the timing of delivery, which will affect perinatal outcomes, is difficult to control $(8,9)$. Although evidence has demonstrated that calcium, aspirin, antioxidants, fish oil, vitamin D and zinc, as well as a number of other substances, play important roles in PE prevention, concrete scientific proof is still lacking $(10,11)$. Previous studies have demonstrated that the formation mechanism of PE is related to placentas (4-11) and its dysfunction is induced by hypoxia (12). In addition, placental development is closely associated with the growth of extravillous cytotrophoblast (EVT) cells (13). Thus, the pathology and therapy of PE needs to be investigated at a cellular level.

A previous study indicated that oxidative stress induced by hypoxia can affect EVT growth through hypoxia-inducible factor (HIF) response (14). The HIF transcription factor family (HIF- $\alpha$ and HIF- $\beta$ ) plays pivotal roles in cellular adaptation, cell migration and signaling under normoxia. HIF-1 $\alpha, \mathrm{HIF}-2 \alpha$ and HIF-3 $\alpha$ are three paralogs of HIF- $\alpha$ in humans (15). HIF- $1 \alpha$ and HIF- $2 \alpha$ are activated in hypoxia and their activities regulate trophoblastic transcription and promote trophoblast invasion (16). It has been hypothesized that during 
hypoxia, increase of HIF-1 $\alpha$ and decrease of placental growth factor (an angiogenic protein) in serum and placentas of pregnant women contributes to PE development (17). In addition, HIF- $2 \alpha$ has been revealed to mediate the expression of Fms like tyrosine kinase receptor (Flt) 1, an angiogenic factor, in placental trophoblasts exposed to hypoxia (18). However, studies conducted on the effects of HIF-3 $\alpha$ on EVT are limited.

The Janus kinase signal transducer and activator of transcription (JAK/STAT) signaling pathway is involved in a number of fundamental biological processes, including cell apoptosis, proliferation and inflammation, and it plays an important role in regulating metabolic homeostasis (19). The JAK/STAT pathway has also been revealed to be associated with implantation between trophoblast cells and receptive endometrium when human chorionic gonadotropin secretion is reduced (20). The intercommunication between the JAK/STAT pathway and ERK1/2 has been demonstrated to promote the invasion of trophoblast cells (21). However, whether HIF-3 $\alpha$ is associated with the JAK/STAT signaling pathway in EVT remains unclear.

The present study is the first, to the best of the authors' knowledge, demonstrating that the function of HIF-3 $\alpha$ in PE development was realized by mediating EVT migration and invasion via the Flt-1/JAK/STAT pathways. The findings of the present study improved the current understanding on the pathological mechanism of PE and provided a novel treatment possibility for PE patients.

\section{Materials and methods}

Clinical specimen collection. Between December 2017 and December 2018, a total of 80 pregnant women (40 normal controls and 40 severe PE patients) who attended the Affiliated Yantai Yuhuangding Hospital of Qingdao University were enrolled. PE was diagnosed according to a previous study (4) as follows: Systolic blood pressure $\geq 140 \mathrm{~mm} \mathrm{Hg}$ or diastolic blood pressure $\geq 90 \mathrm{~mm} \mathrm{Hg}$ on 2 separate readings in gravidae, combined with proteinuria $>300 \mathrm{mg}$ per day. None of the research subjects had pregnancy complications, such as heart disease or chronic nephritis merger, and their pregnancy was a singleton. The following data were collected: Maternal age at delivery, numbers of primigravidae, gestation, body mass index (BMI) of pregnant women, systolic pressure, diastolic pressure, 24-h urinary protein and delivery mode. No significant difference between the two groups of subjects in age or BMI was observed. All patients had signed informed consent and agreed that their placentas would be used for clinical research. The clinical trial program had been reviewed and approved by the Ethics Committee of the Affiliated Yantai Yuhuangding Hospital of Qingdao University (YYQ2019012716). The samples obtained were flash-frozen in liquid nitrogen and stored at $-80^{\circ} \mathrm{C}$ prior to the experiments.

Cell culture and cell treatment. Human EVT HTR8/SVneo cell line was purchased from American Type Culture Collection and cultured in high-glucose Dulbecco's modified Eagle's medium supplemented with $10 \%$ fetal bovine serum (both from Gibco; Thermo Fisher Scientific, Inc.), $50 \mu \mathrm{g} / \mathrm{ml}$ penicillin and $50 \mu \mathrm{g} / \mathrm{ml}$ streptomycin (Target Molecule Corp.). Under normoxia culture (Control), the cells were incubated in a humid incubator at $37^{\circ} \mathrm{C}$ with $5 \% \mathrm{CO}_{2}$. For hypoxia, the cells were cultured at $37^{\circ} \mathrm{C}$ in an incubator with a gas mixture of $5 \% \mathrm{CO}_{2}, 2 \% \mathrm{O}_{2}$ and $93 \% \mathrm{~N}_{2}$ for
$12,24,48$ or $72 \mathrm{~h}$. In addition, HTR8/SVneo cells cultured under hypoxia were treated by $10 \mathrm{mmol} / 1$ AG490 (cat. no. A4139; APExBIO) for $12 \mathrm{~h}$ at $37^{\circ} \mathrm{C}$ to inhibit the phosphorylation of the JAK/STAT signaling pathway of the cells cultured under hypoxia. Cells without any treatment served as Control.

Cell transfection. The cells $\left(10^{4}\right.$ cells/well) were cultured to a confluence of $90 \%$ in 6-well plates before cell transfection. Opti-MEM $^{\text {тм }}$ (Invitrogen; Thermo Fisher Scientific, Inc.) was used to dilute Lipofectamine ${ }^{\circledR} 2000$ transfection reagent (Invitrogen; Thermo Fisher Scientific, Inc.) and $2.5 \mu \mathrm{g}$ DNA of HIF-3 $\alpha$-pcDNA3.1 combinant vector (HIF-3 $\alpha$ group) or pcDNA3.1 empty vector (cat. no. sc-403487-HDR, Santa Cruz Biotechnology, Inc.; NC group). The mixture of diluted DNA and diluted Lipofectamine ${ }^{\circledR} 2000$ (1:1 ratio) was incubated for $5 \mathrm{~min}$ at room temperature and then added into each well to co-incubate with the cells at $37^{\circ} \mathrm{C}$ for $48 \mathrm{~h}$. Finally, transfection efficiency was detected by reverse transcription-quantitative (RT-q) PCR. Cells without any treatment served as Control.

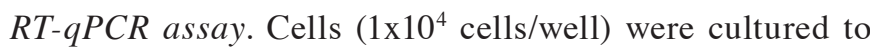
$90 \%$ confluence in 6 -well plates before RNA extraction. Total RNA was extracted from the placental tissues and HTR8/SVneo cells using TRIzol ${ }^{\text {TM }}$ Reagent (Invitrogen; Thermo Fisher Scientific, Inc.) and the RNA concentration was detected by DR6000 ${ }^{\mathrm{TM}}$ UV VIS Spectrophotometer (HACH) at a wavelength of $280 \mathrm{~nm}$. The first strand of cDNAs was synthesized using TaqMan ${ }^{\mathrm{TM}}$ Reverse Transcription Reagents (cat. no. 4304134; Thermo Fisher Scientific, Inc.) according to the manufacturer's instructions and then subjected to real-time PCR amplification operated with TaqMan ${ }^{\mathrm{TM}}$ Universal PCR Master Mix (cat. no. 4326708; Thermo Fisher Scientific, Inc.) in the PCR QuantStudio ${ }^{\mathrm{TM}} 3$ Real-Time PCR System (Thermo Fisher Scientific, Inc.). The primers used were as follows: HIF-3 $\alpha$ forward, 5'-GCACCCTCAACCTCAAGG C-3' and reverse, 5'-GCAATCCTGTCGTCACAGTAG-3'; Flt-1 forward, 5'-TGCCGGGTTACGTCACCTA-3' and reverse, 5'-GTCCCAGATTATGCGTTTTCCAT-3'; GAPDH forward, 5'-CCATCTTCCAGGAGCGAGAT-3' and reverse, 5'-TGCTGATGATCTTGAGGCTG-3' (reverse). The conditions were set as follows: At $50^{\circ} \mathrm{C}$ for $2 \mathrm{~min}$, then at $95^{\circ} \mathrm{C}$ for $10 \mathrm{~min}$ and 40 cycles at $95^{\circ} \mathrm{C}$ for $15 \mathrm{sec}$ and at $60^{\circ} \mathrm{C}$ for $1 \mathrm{~min}$. The relative expression of each mRNA was calculated by comparative quantification cycle (Cq) method $2^{-\Delta \Delta \mathrm{Cq}}(22)$.

Western blot analysis. The protein expression levels of the treated HTR8/SVneo cells were detected by western blot analysis. Total proteins of the HTR8/SVneo cells were isolated by ProteoPrep ${ }^{\circledR}$ Total Extraction Sample kit (EMD Millipore) according to the manufacturer's instructions and protein concentration was detected by Modified BCA Protein Assay kit (Sangon Biotech Co., Ltd.) in DR6000 ${ }^{\mathrm{TM}}$ UV VIS Spectrophotometer at a wavelength of $562 \mathrm{~nm}$. Next, $50 \mu \mathrm{g}$ of protein samples were separated on $10 \%$ sodium dodecyl sulfate-polyacrylamide gel electrophoresis (SDS-PAGE; Beijing Solarbio Science \& Technology Co., Ltd.) and then transferred on polyvinylidene fluoride membranes. The membranes were blocked by $5 \%$ non-fat dry milk at $37^{\circ} \mathrm{C}$ for $1 \mathrm{~h}$ and incubated together with primary antibodies HIF-3 $\alpha$ (1:500; product code ab10134; Abcam), Flt-1 (1:1,000; product code ab2350), phosphorylated (p-)JAK2 (1:2,000; 
Table I. Clinical data of normal and PE pregnant women.

\begin{tabular}{lcc}
\hline Variable & Normal, $\mathrm{n}=40$ & PE, $\mathrm{n}=40$ \\
\hline Maternal age (years) & $28.65 \pm 4.82$ & $30.14 \pm 6.12$ \\
Primigravida (n) & 31 & $17^{\mathrm{a}}$ \\
Gestation (weeks) & $39.35 \pm 1.41$ & $32.46 \pm 3.54^{\mathrm{a}}$ \\
BMI (at delivery) & $27.49 \pm 5.43$ & $33.81 \pm 5.82^{\mathrm{a}}$ \\
Systolic pressure (mm Hg) & $113.24 \pm 8.71$ & $163.48 \pm 21.57^{\mathrm{a}}$ \\
Diastolic pressure (mm Hg) & $63.73 \pm 7.47$ & $107.12 \pm 13.34^{\mathrm{a}}$ \\
Urinary protein in 24 h (g) & $0.21 \pm 0.06$ & $5.82 \pm 1.73^{\mathrm{a}}$ \\
Delivery method (vaginal delivery/cesarean section) & $27 / 13$ & $5 / 35^{\mathrm{a}}$ \\
\hline
\end{tabular}

The chi-square test was applied for clinical sample analysis. ${ }^{\mathrm{P}}<0.001, \mathrm{PE}$ vs. normal group. PE, preeclamptic; BMI, body mass index.

product code ab32101), JAK2 (1:5,000; product code ab108596), p-STAT3 (1:5,000; product code ab76315), STAT3 (1:5,000; product code ab119352) and GAPDH (1:20,000; product code ab8245; all from Abcam) at $4^{\circ} \mathrm{C}$ overnight. GAPDH served as an internal reference. After washing the membranes with phosphate-buffered solution three times, the membranes were further incubated with corresponding secondary antibodies goat anti-rabbit IgG H\&L (HRP) (1:2,000; product code ab205718) and goat anti-mouse IgG H\&L (HRP) $(1: 2,000$; product code ab205719; both from Abcam) at $37^{\circ} \mathrm{C}$ for $1 \mathrm{~h}$. Finally, the different protein brands were recorded and analyzed with Bio-Rad ChemiDoc $^{\mathrm{TM}}$ XRS+ System (Bio-Rad Laboratories, Inc.) with Image Lab software (version 4.1; Bio-Rad Laboratories, Inc.).

Cell viability. Cell viability of the treated HTR8/SVneo cells was measured by MTT assay with Cell Proliferation Kit I (Roche Diagnostics). MTT reagent $(0.5 \mathrm{mg} / \mathrm{ml} ; 10 \mu \mathrm{l})$ was added to each well and incubated at $37^{\circ} \mathrm{C}$ for $4 \mathrm{~h}$. Next, optical density was recorded using a microplate reader (Bio-Rad Laboratories, Inc.) at a wavelength of $495 \mathrm{~nm}$.

Flow cytometry. Cell apoptosis was detected by performing flow cytometry using an Annexin V-FITC Apoptosis Detection kit (Sigma-Aldrich; Merck KGaA) following the manufacturer's protocol. HTR8/SVneo cells treated by HIF-3 $\alpha$ and/or AG490 were suspended in buffer solution containing Annexin V-FITC and propidium iodide $(50 \mathrm{mg} / \mathrm{ml})$ at $37^{\circ} \mathrm{C}$ for $1 \mathrm{~h}$ in the dark. Then the flow cytometer CytoFLEX (Beckman Coulter, Inc.) and CytExpert software (version 4.1; Beckman Coulter, Inc.) was used for analysis.

Statistical analysis. All the data were presented as the mean \pm standard deviation and analyzed using SPSS software (version 13.0; SPSS, Inc.). Chi-square test was applied for clinical sample analysis in Table I. Statistical differences between groups were compared using the Student's t-test or one-way analysis of variance (ANOVA) followed by Tukey's t-test. $\mathrm{P}<0.05$ was considered to indicate a statistically significant difference.

\section{Results}

Clinical data from normal and PE pregnant women. Clinical data, including maternal age, the number of primigravida, gestation before delivery, BMI at delivery, blood pressure, urine protein and delivery mode, were collected from PE patients and normal controls and are listed in Table I. The maternal age range in the PE group ( $30.14 \pm 6.12$ years old) was higher than the normal group (28.65 \pm 4.82 years old). In addition, compared with the PE group, primigravidae accounted for the majority of the subjects in the normal group. Generally, normal pregnant women had a longer gestation period but had a lower BMI than PE pregnant patients. In addition, systolic blood pressures, diastolic blood pressures and 24-h urine protein were significantly higher among patients in the PE group than normal controls. In addition, most of the PE gravidae underwent a cesarean section, whereas normal pregnant women commonly delivered vaginally.

Downregulation of HIF-3 $\alpha$ and Flt-1 in PE patients. The mRNA expression levels of $H I F-3 \alpha$ and Flt-1 were detected by performing RT-qPCR. Compared with the normal group, the mRNA expression levels of $H I F-3 \alpha(\mathrm{P}<0.001$; Fig. 1A) and Flt-1 (P<0.001; Fig. 1D) were significantly reduced in the PE group. Western blot analysis was performed for the detection of the expression levels of relative proteins and the results demonstrated that the expression levels of the HIF-3 $\alpha$ protein of $\mathrm{PE}$ pregnant woman were decreased compared with those in normal pregnant women $(\mathrm{P}<0.05$; Fig. $1 \mathrm{~B}$ and $\mathrm{C})$ and that the relative Flt-1 expression level of the $\mathrm{PE}$ group was reduced compared with the normal group $(\mathrm{P}<0.05$; Fig. 1E and $\mathrm{F})$.

Hypoxia affects HIF-3 $\alpha$ and Flt-1 expression levels, the JAK/STAT3 signaling pathway, cell viability and cell apoptosis of HTR8/SVneo cells. HTR8/SVneo cells were cultured under hypoxic conditions for 12, 24, 48 and $72 \mathrm{~h}$ and the protein expression levels were assessed by western blot analysis. The protein levels of HIF-3 $\alpha$ and Flt-1 of HTR8/SVneo cells were markedly increased at $12 \mathrm{~h}$ but reduced after $12 \mathrm{~h}$. After 72-h culture in hypoxia, HIF-3 $\alpha$ and Flt-1 protein expression levels were even lower than those in normoxia $(\mathrm{P}<0.05$; Fig. $2 \mathrm{~A}$ and $\mathrm{B})$. Next, RT-qPCR was conducted to detect the relative mRNA expression levels. Similarly, in comparison with the normoxia group, the mRNA expression levels of HIF-3 $\alpha$ and Flt-1 were first increased at $12 \mathrm{~h}$ and then reduced after $12 \mathrm{~h}(\mathrm{P}<0.05$; Fig. 2C). The protein expression levels of the JAK/STAT signaling pathway were detected by western blot analysis and the data demonstrated that the relative protein expression levels 


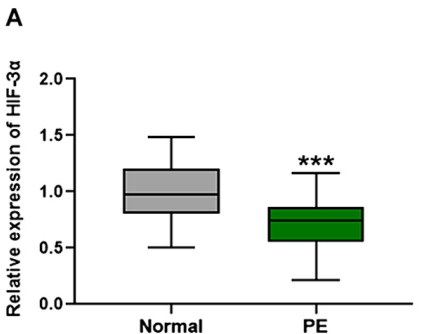

B

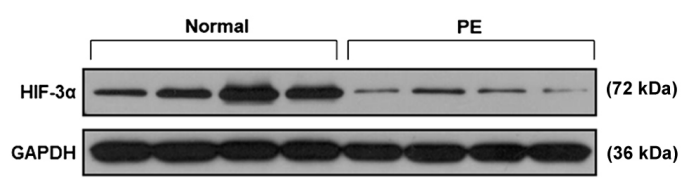

D

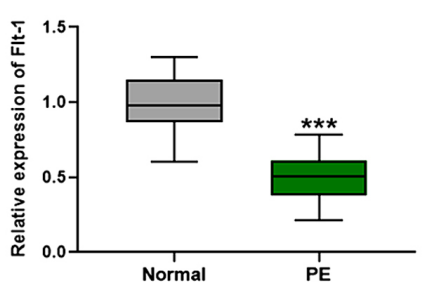

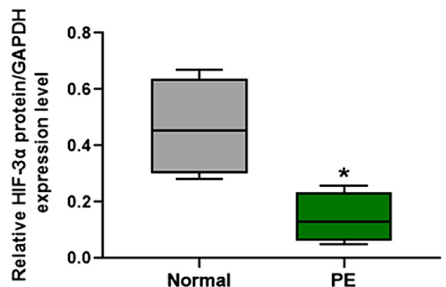
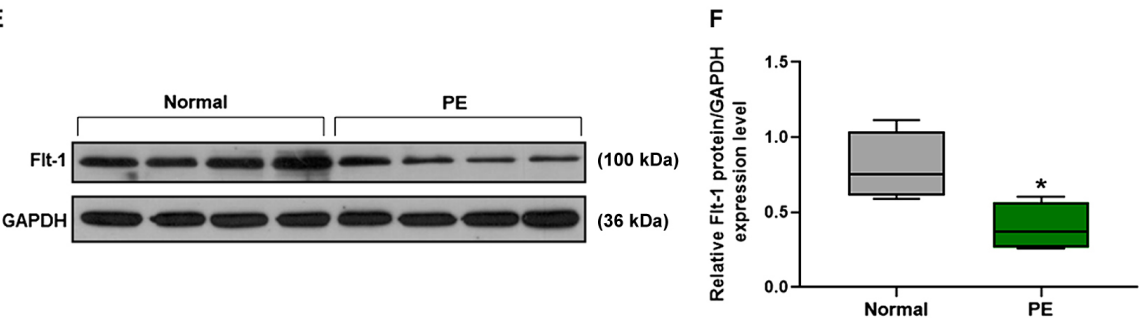

Figure 1. The gene expression levels of HIF-3 $\alpha$ and Flt-1 are reduced in PE patients compared with normal controls. In total, 40 normal placentas and 40 PE placentas were used as the experimental samples. RT-qPCR was performed to assess the expression levels of (A) $H I F-3 \alpha$ and (D) Flt-1. Then the relative protein expression levels of (B and C) HIF-3 $\alpha$ and ( $\mathrm{E}$ and F) Flt-1 were detected by western blot analysis. ${ }^{*} \mathrm{P}<0.05$ and ${ }^{* * *} \mathrm{P}<0.001$ vs. normal controls, $\mathrm{n}=3$. $\mathrm{PE}$, preeclampsia; RT-qPCR, reverse transcription-quantitative PCR; HIF, hypoxia-inducible factor; Flt, Fms-like tyrosine kinase receptor; Normal, normal controls.

A

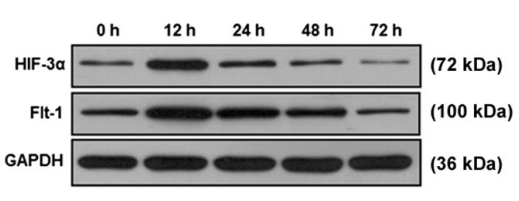

D

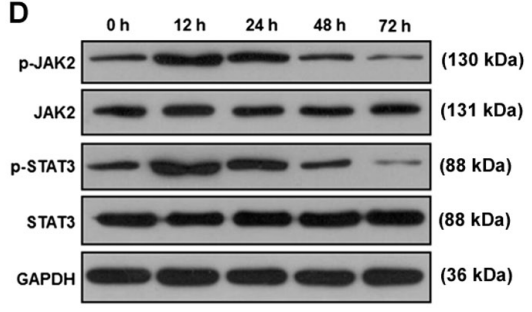

$\mathbf{F}$

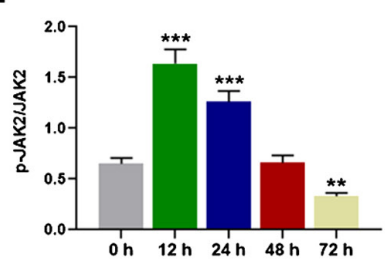

B

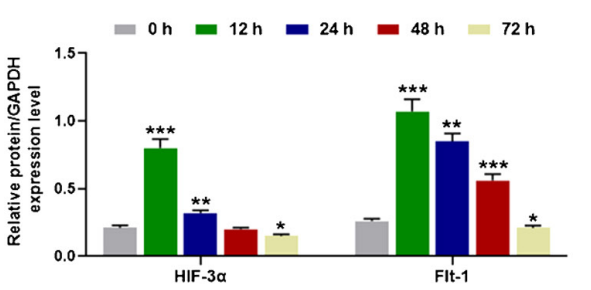

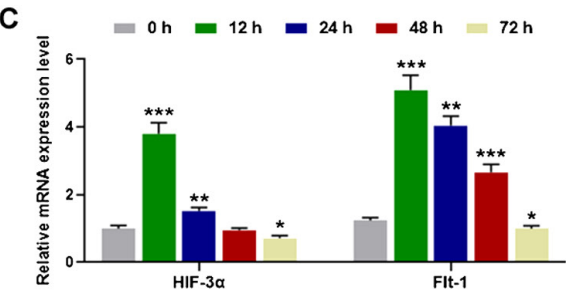

E

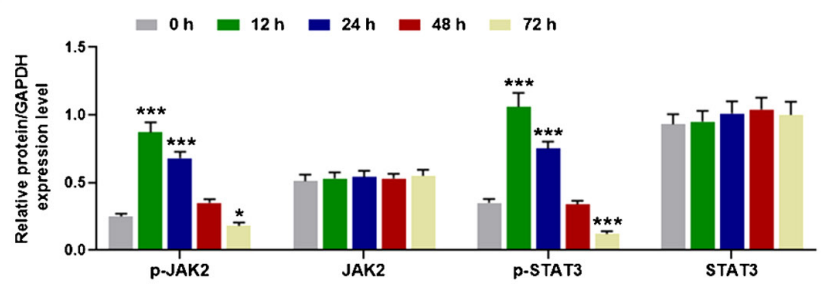

H

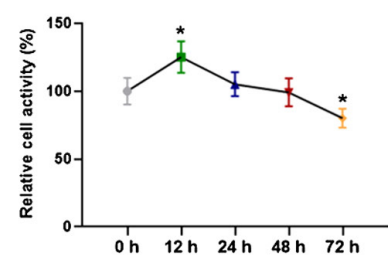

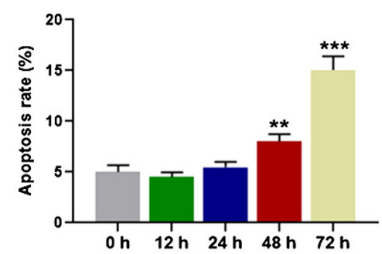

$\mathbf{J}$

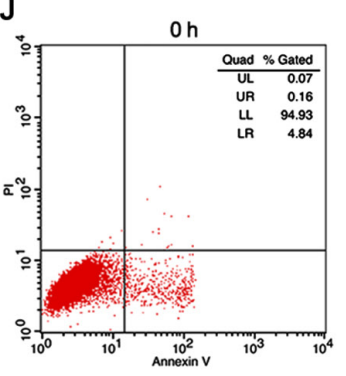

G

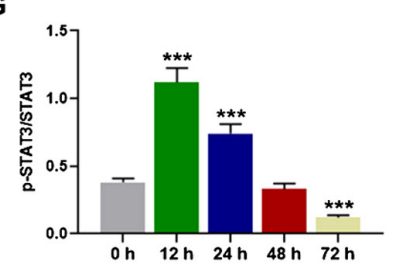

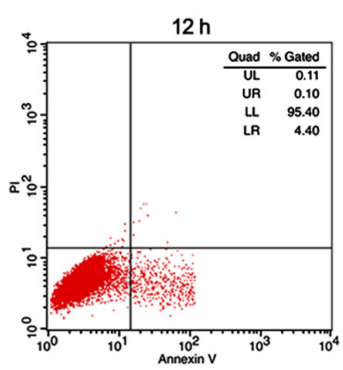
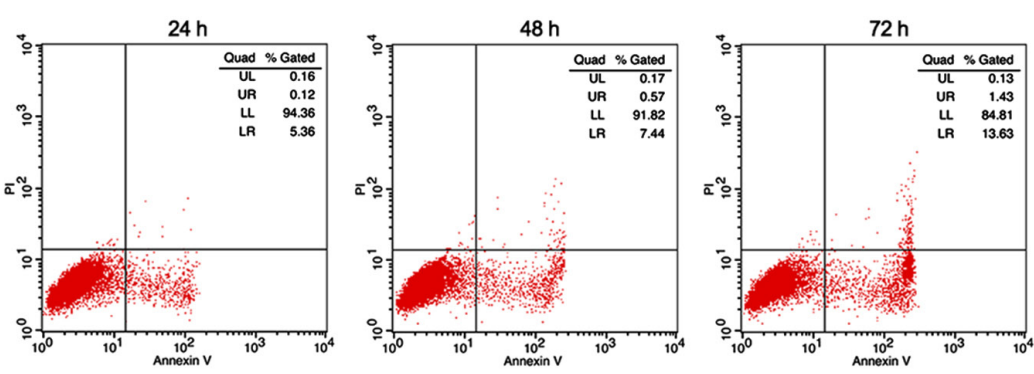

Figure 2. Hypoxia affects HIF-3 $\alpha$ and Flt-1 expression levels, JAK2 and STAT3 phosphorylation, and cell viability and cell apoptosis of HTR8/SVneo cells. (A and B) Western blot analysis detected the protein expression levels of HIF-3 $\alpha$ and Flt-1, followed by (C) RT-qPCR to assess the mRNA expression levels. (D-G) The protein expression levels of p-JAK2, JAK2, p-STAT3, STAT3, p-JAK2/JAK2 and p-STAT3/STAT3 were detected by western blot analysis. (H) Cell activity was detected by MTT assay and (I and J) cell apoptosis was detected by flow cytometry. ${ }^{*} \mathrm{P}<0.05,{ }^{* *} \mathrm{P}<0.01$ and ${ }^{* * * *} \mathrm{P}<0.001, \mathrm{vs} .0 \mathrm{~h}, \mathrm{n}=3$. HIF, hypoxia-inducible factor; Flt, Fms like tyrosine kinase receptor; JAK, Janus kinase; STAT, signal transducer and activator of transcription; RT-qPCR, reverse transcription-quantitative PCR; p-, phosphorylated. 
A

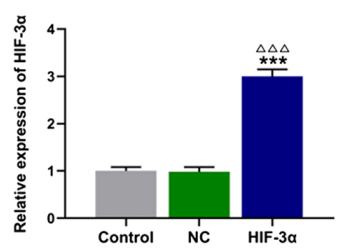

B

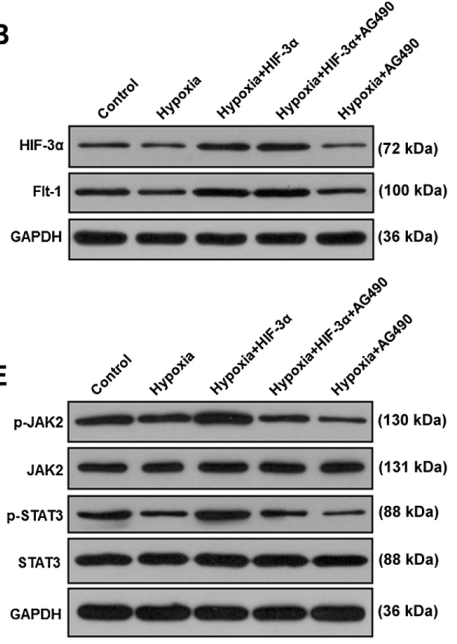

C

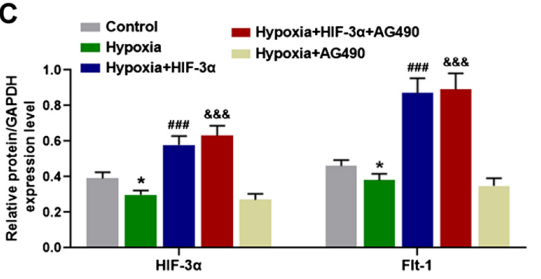

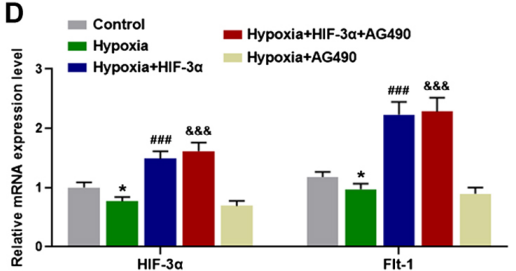

$\mathbf{F}$

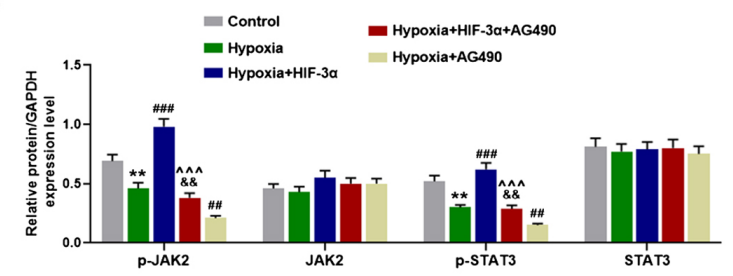

G

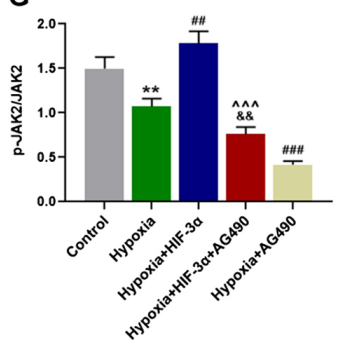

H

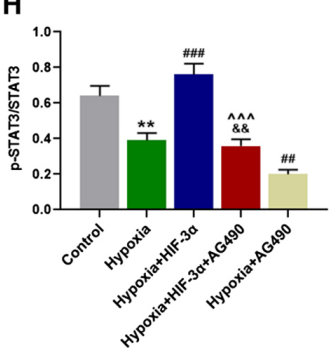

I

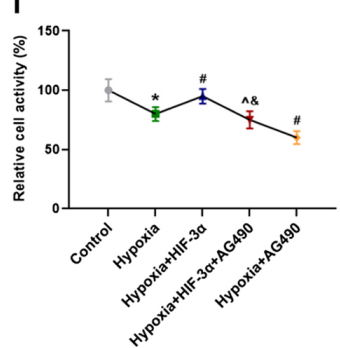

$J$

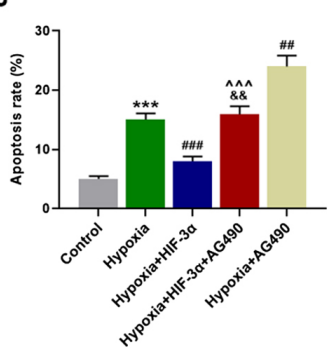

K
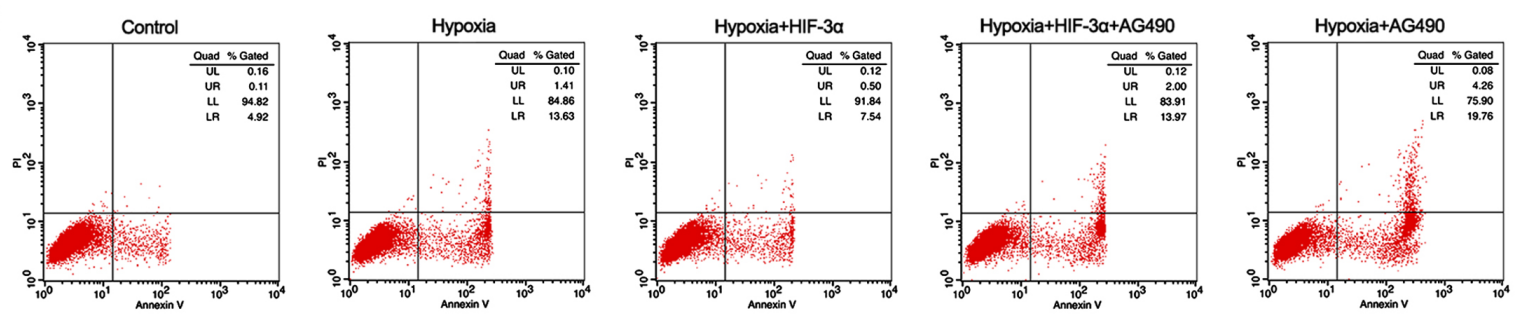

Figure 3. HIF-3 $\alpha$ regulates cell proliferation and cell apoptosis via upregulation of the JAK/STAT signaling pathway of HTR8/SVneo cells. (A) The transfection efficiency was detected by RT-qPCR and HIF-3 $\alpha$ was significantly overexpressed in the HIF-3 $\alpha$ group. Cells cultured in hypoxia for $12 \mathrm{~h}$ were transfected with HIF-3 $\alpha$ and AG490 alone or in combination, then (B and C) western blot assays were used to detect the protein expression levels of HIF-3 $\alpha$ and Flt-1. (D) RT-qPCR was performed to measure HIF-3 $\alpha$ and Flt-1 mRNA expression levels. (E-H) Phosphorylation of the JAK/STAT signaling pathway of the treated cells was detected by western blot analysis (the expression levels of p-JAK2, JAK2, p-STAT3, STAT3, p-JAK2/JAK2 and p-STAT3/STAT3). (I) Cell viability of corresponding treated cells was detected by MTT, followed by ( $\mathrm{J}$ and $\mathrm{K}$ ) flow cytometry for the detection of cell apoptosis. ${ }^{*} \mathrm{P}<0.05,{ }^{* *} \mathrm{P}<0.01$ and ${ }^{* * * *} \mathrm{P}<0.001 \mathrm{vs}$. the Control; ${ }^{\Delta \Delta \Delta} \mathrm{P}<0.001 \mathrm{vs}$. NC; ${ }^{*} \mathrm{P}<0.05$, ${ }^{\# \#} \mathrm{P}<0.01$ and ${ }^{\# \# !} \mathrm{P}<0.001$, vs. Hypoxia; : $\mathrm{P}<0.05$, \&\& $\mathrm{P}<0.01$ and ${ }^{\& \& \&} \mathrm{P}<0.001$ vs. Hypoxia+AG490; ${ }^{\wedge} \mathrm{P}<0.05$ and ${ }^{\wedge \wedge} \mathrm{P}<0.001$ vs. Hypoxia+HIF-3 $\alpha, \mathrm{n}=3$. HIF, hypoxia-inducible factor; JAK, Janus kinase; STAT, signal transducer and activator of transcription; RT-qPCR, reverse transcription-quantitative PCR; p-, phosphorylated.

of p-JAK2 and p-STAT3 were significantly promoted at 12 and $24 \mathrm{~h}$, but were suppressed at $72 \mathrm{~h}$, compared with the normoxia group, and the protein expression levels of JAK2 and STAT3 remained unchanged over time $(\mathrm{P}<0.05$; Fig. $2 \mathrm{D}$ and $\mathrm{E})$. Thus, in hypoxia, p-JAK2/JAK2 and p-STAT3/STAT3 were significantly increased at 12 and $24 \mathrm{~h}$, but reduced at $72 \mathrm{~h}$ compared with those in the normoxia group $(\mathrm{P}<0.01$; Fig. $2 \mathrm{~F}$ and $\mathrm{G})$. Then cell viability of HTR8/SVneo cells was detected by MTT and the data demonstrated that cell activity was markedly promoted at $12 \mathrm{~h}$ in hypoxia, but inhibited at $72 \mathrm{~h}$ compared with the normoxia group $(\mathrm{P}<0.05$; Fig. $2 \mathrm{H})$. Finally, flow cytometry was performed to detect cell apoptosis; HTR8/SVneo cell apoptosis was not altered at 12 and $24 \mathrm{~h}$, but was significantly increased at 48 and $72 \mathrm{~h}$ in hypoxia $(\mathrm{P}<0.01$; Fig. $2 \mathrm{I}$ and $\mathrm{J})$.

HIF-3 $\alpha$ regulates cell proliferation and cell apoptosis via upregulation of the JAK/STAT signaling pathway of HTR8/SVneo cells. The treated HTR8/SVneo cells were cultured in hypoxia for $12 \mathrm{~h}$ and then subjected to western blot analysis and RT-qPCR. The transfection efficiency was detected by RT-qPCR and $H I F-3 \alpha$ was significantly over-expressed in the HIF-3 $\alpha$ group $(\mathrm{P}<0.01$; Fig. $3 \mathrm{~A})$. Compared with the control 
group, hypoxia significantly reduced the protein and mRNA levels of HIF-3 $\alpha$ and Flt-1, which were reversed by HIF-3 $\alpha$ overexpression. In addition, no significant effect of AG490 on HIF-3 $\alpha$ and Flt-1 levels was observed compared with the hypoxia + HIF-3 $\alpha$ group ( $\mathrm{P}<0.05$; Fig. 3B-D). Phosphorylation of the JAK/STAT signaling pathway was assessed by western blot analysis and the data revealed that chronic hypoxia and AG490 markedly inhibited the protein expression levels of p-JAK2 and p-STAT3. However, upregulation of HIF-3 $\alpha$ expression significantly increased the phosphorylation of the JAK/STAT signaling pathway by antagonizing the effect of hypoxia $(\mathrm{P}<0.01$; Fig. 3E-H). According to the results of the MTT assay, viability of HTR8/SVneo cells was suppressed by chronic hypoxia and AG490, but was promoted by the increase of anti-hypoxic HIF-3 $\alpha$ ( $\mathrm{P}<0.05$; Fig. 3I). Flow cytometry was performed to assess cell apoptosis of HTR8/SVneo cells. After 72-h culture in hypoxia and treatment with AG490, cell apoptosis of HTR8/SVneo cells was significantly increased, however, overexpression of HIF-3 $\alpha$ abrogated the effects of hypoxia on HTR8/SVneo cells ( $\mathrm{P}<0.01$; Fig. 3J and K).

\section{Discussion}

In the transcriptional response to hypoxia, the HIF family plays key roles in the process and HIF- $1 \alpha$ and HIF- $2 \alpha$ are two master regulators (15-18). Although the third paralog of HIF- $\alpha$, which is oxygen-dependent HIF- $3 \alpha$, can also activate transcriptional responses to hypoxia and mediate the hypoxia-induced development retardation, little is known about HIF-3 $\alpha$ (23). The present study compared the protein and mRNA expression levels of HIF- $3 \alpha$ extracted from normal and PE placental tissues. The results demonstrated that HIF-3 $\alpha$ gene expression levels of the PE group were markedly reduced, indicating that the change of HIF-3 $\alpha$ gene expression levels was related to PE. Flt-1 plays a crucial role in angiopoiesis (24) and is associated with placental degeneration and invasion (25). Yamashita et al (25) demonstrated that the sFLT-1 (an isoform of Flt-1) expression level is notably high in patients with placenta previa and the study also hypothesized that PE may be correlated with placenta previa. In the present study, the gene expression of Flt-1 in PE samples and normal samples was determined and the data revealed that Flt-1 expression of PE tissues was reduced. A previous study demonstrated that suppressing HIF-2 $\alpha$ can inhibit hypoxia-induced upregulation of Flt-1 expression of cytotrophoblasts (18). However, whether HIF-3 $\alpha$ can regulate Flt-1 expression of EVT was not clarified. After the transfection of HIF-3 $\alpha$ into HTR8/SVneo cells in hypoxia, the present study observed that overexpression of HIF- $3 \alpha$ resulted in the upregulation of Flt-1, indicating that upregulated HIF-3 $\alpha$ had regulatory effects on Flt-1 expression.

It has previously been revealed that in hypoxia, HIF-3 $\alpha$ protein and mRNA levels are increased in cultured human lung epithelial cells based on the protein stability and transcriptional activation (26). In addition, HIF-3 $\alpha$ expression was increased in the Caki-1 renal carcinoma cells in response to hypoxia treatment (27). However, in the present study, HTR8/SVneo cells were cultured in hypoxia and the gene expression of HIF-3 $\alpha$ was significantly increased at $12 \mathrm{~h}$ but reduced after 12 h. At 72 h, HIF-3 $\alpha$ and Flt-1 gene expression levels were inhibited compared with the normoxia group. With regard to Flt-1, it has been demonstrated that the overexpression of soluble Flt-1 exhibited a cytotoxic effect on BeWo choriocarcinoma cells (28). Flt-1 has been revealed to be expressed at a higher level in advanced colorectal cancer cells compared with localized ones and to play an important role in colorectal cancer progression (29). However, the present study found that in hypoxia, Flt-1 gene expression was increased at $12 \mathrm{~h}$ and then reduced.

JAK proteins are activated by intracellular receptors through autophosphorylation, then further phosphorylate and promote STAT proteins; JAK in combination with STAT proteins creates a high degree of specificity (19). For instance, the inhibition of JAK1 and JAK2 activity reduced STAT3 phosphorylation and translocation in prostatic cancer cells (30); inhibiting miR-210 resulted in the promotion of cell apoptosis and the suppression of cell proliferation of vascular endothelial cells via blocking of the JAK/STAT signaling pathway (31); the activation of the JAK/STAT signaling pathway contributed to the proliferation, migration and invasion of glioma cells (32). Nevertheless, in the present study, p-JAK2 and p-STAT3 protein expression levels were first increased at $12 \mathrm{~h}$ and then reduced at $72 \mathrm{~h}$ in hypoxia compared with in normoxia and in addition, the cell viability of HTR8/SVneo cells fluctuated with phosphorylation of the JAK/STAT signaling pathway, while cell apoptosis of EVT was steadily increased over time.

To prevent the JAK/STAT signaling pathway from phosphorylation, AG490 was used to inhibit the activities of JAK2 and STAT3 according to previous studies $(32,33)$. HTR8/SVneo cells were transfected with HIF- $3 \alpha$ and AG490 alone or in combination and cultured in hypoxia. Chronic hypoxia culture (72-h hypoxia) significantly reduced the protein and mRNA expression levels of HIF-3 $\alpha$ and Flt-1 as well as the expression levels of p-JAK2 and p-STAT3, indicating that the phosphorylation of JAK/STAT was inhibited. AG490 did not affect HIF-3 $\alpha$ and Flt-1 gene expression levels, but it markedly inhibited the protein expression levels of p-JAK2 and p-STAT3. By upregulating HIF-3 $\alpha$ expression levels in vitro, the HIF-3 $\alpha$ and Flt-1 expression levels were markedly increased and phosphorylation of JAK/STAT was promoted, suggesting that the abilities of migration and invasion of HTR8/SVneo cells were increased. In addition, AG490-reduced viability and AG490-promoted apoptosis in chronic hypoxia were markedly abrogated by the upregulation of HIF-3 $\alpha$ expression. All these results indicated that HIF-3 $\alpha$ overexpression had regulatory effects on the Flt-1/JAK/STAT pathway in EVT HTR8/SVneo cells.

In conclusion, HIF-3 $\alpha$ may be a regulator in the growth of EVT HTR8/SVneo cells in hypoxia. Upregulation of HIF-3 $\alpha$ promoted the Flt-1 expression and phosphorylation levels of JAK2 and STAT3. In addition, the effects of hypoxia on inhibiting cell viability and promoting cell apoptosis were reversed to a large extent. Thus, the present study concluded that HIF-3 $\alpha$ affected PE development by regulating EVT growth via upregulation of the Flt-1/JAK/STAT signaling pathway in hypoxia. The limitation of the present study was the small clinical sample size, which should be improved in future research. 


\section{Acknowledgements}

Not applicable.

\section{Funding}

No funding was received.

\section{Availability of data and materials}

The datasets used and/or analyzed during the current study are available from the corresponding author on reasonable request.

\section{Authors' contributions}

HQ, QY and BJ made substantial contributions to the conception and design of the study. WZ, YZ and LM performed data acquisition, data analysis and interpretation. HQ, QY and BJ drafted the article or critically revised it for important intellectual content. All authors agreed to be accountable for all aspects of the work in ensuring that questions related to the accuracy or integrity of the work are appropriately investigated and resolved. All authors read and approved the final manuscript.

\section{Ethics approval and consent to participate}

All procedures performed in studies involving human participants were in accordance with the ethical standards of the institutional and/or national research committee and with the 1964 Helsinki declaration and its later amendments or comparable ethical standards. All patients had signed informed consent and agreed that their placentas would be used for clinical research. The clinical trial program had been reviewed and approved by the Ethics Committee of the Affiliated Yantai Yuhuangding Hospital of Qingdao University (YYQ2019012716).

\section{Patient consent for publication}

Not applicable.

\section{Competing interests}

The authors declare that they have no competing interests.

\section{References}

1. Karumanchi SA and Granger JP: Preeclampsia and pregnancy-related hypertensive disorders. Hypertension 67: 238-242, 2016.

2. El-Sayed AAF: Preeclampsia: A review of the pathogenesis and possible management strategies based on its pathophysiological derangements. Taiwan J Obstet Gynecol 56: 593-598, 2017.

3. Park JL, Lee SW, Oh KJ and Hong JS: High mean blood pressure during the first trimester is predictive of future preeclampsia development in healthy pregnant women: A cohort study in Korea. Clin Exp Obstet Gynecol 46: 770-775, 2019.

4. Mol BWJ, Roberts CT, Thangaratinam S, Magee LA, de Groot CJM and Hofmeyr GJ: Pre-eclampsia. Lancet 387: 999-1011, 2016.

5. Morton A: Imitators of preeclampsia: A review. Pregnancy Hypertens 6: 1-9, 2016.
6. Ramos JGL, Sass N and Costa SHM: Preeclampsia. Rev Bras Ginecol Obstet 39: 496-512, 2017.

7. Malik A, Jee B and Gupta SK: Preeclampsia: Disease biology and burden, its management strategies with reference to India. Pregnancy Hypertens 15: 23-31, 2019.

8. Bokslag A, van Weissenbruch M, Mol BW and de Groot CJ: Preeclampsia; short and long-term consequences for mother and neonate. Early Hum Dev 102: 47-50, 2016.

9. Steegers EA, von Dadelszen P, Duvekot JJ and Pijnenborg R: Pre-eclampsia. Lancet 376: 631-644, 2010.

10. Atallah A, Lecarpentier E, Goffinet F, Doret-Dion M, Gaucherand $\mathrm{P}$ and Tsatsaris V: Aspirin for prevention of preeclampsia. Drugs 77: 1819-1831, 2017.

11. Correa PJ, Palmeiro Y, Soto MJ, Ugarte C and Illanes SE: Etiopathogenesis, prediction, and prevention of preeclampsia. Hypertens Pregnancy 35: 280-294, 2016.

12. van Patot MC, Ebensperger G, Gassmann M and Llanos AJ: The hypoxic placenta. High Alt Med Biol 13: 176-184, 2012.

13. Gude NM, Roberts CT, Kalionis B and King RG: Growth and function of the normal human placenta. Thromb Res 114: 397-407, 2004

14. Macklin PS, McAuliffe J, Pugh CW and Yamamoto A: Hypoxia and HIF pathway in cancer and the placenta. Placenta 56: 8-13, 2017.

15. Graham AM and Presnell JS: Hypoxia Inducible Factor (HIF) transcription factor family expansion, diversification, divergence and selection in eukaryotes. PLoS One 12: e0179545, 2017.

16. Highet AR, Khoda SM, Buckberry S, Leemaqz S, BiancoMiotto T, Harrington E, Ricciardelli C and Roberts CT: Hypoxia induced $\mathrm{HIF}-1 / \mathrm{HIF}-2$ activity alters trophoblast transcriptional regulation and promotes invasion. Eur J Cell Biol 94: 589-602, 2015.

17. Rath G, Aggarwal R, Jawanjal P, Tripathi R and Batra A: HIF-1 alpha and placental growth factor in pregnancies complicated with preeclampsia: A qualitative and quantitative analysis. J Clin Lab Anal 30: 75-83, 2016.

18. Sasagawa T, Nagamatsu T, Morita K, Mimura N, Iriyama T, Fujii $\mathrm{T}$ and Shibuya M: HIF- $2 \alpha$, but not HIF-1 $\alpha$, mediates hypoxia-induced up-regulation of Flt-1 gene expression in placental trophoblasts. Sci Rep 8: 17375, 2018.

19. Dodington DW, Desai HR and Woo M: JAK/STAT-emerging players in metabolism. Trends Endocrinol Metab 29: 55-65, 2018.

20. Gupta SK, Malhotra SS, Malik A, Verma S and Chaudhary P: Cell signaling pathways involved during invasion and syncytialization of trophoblast cells. Am J Reprod Immunol 75: 361-371, 2016.

21. Malik A, Pal R and Gupta SK: Interdependence of JAK-STAT and MAPK signaling pathways during EGF-mediated HTR-8/SVneo cell invasion. PLoS One 12: e0178269, 2017.

22. Guénin S, Mauriat M, Pelloux J, Van Wuytswinkel O, Bellini C and Gutierrez L: Normalization of qRT-PCR data: The necessity of adopting a systematic, experimental conditions-specific, validation of references. J Exp Bot 60: 487-493, 2009.

23. Zhang P, Yao Q, Lu L, Li Y, Chen PJ and Duan C: Hypoxia-inducible factor 3 is an oxygen-dependent transcription activator and regulates a distinct transcriptional response to hypoxia. Cell Rep 6: 1110-1121, 2014.

24. Chappell JC, Cluceru JG, Nesmith JE, Mouillesseaux KP, Bradley VB, Hartland CM, Hashambhoy-Ramsay YL, Walpole J, Peirce SM, Mac Gabhann F and Bautch VL: Flt-1 (VEGFR-1) coordinates discrete stages of blood vessel formation. Cardiovasc Res 111: 84-93, 2016.

25. Yamashita $M$, Kumasawa K, Nakamura $H$ and Kimura T: Soluble FLT-1 rules placental destiny. Biochem Biophys Res Commun 496: 1243-1249, 2018.

26. Li QF, Wang XR, Yang YW and Lin H: Hypoxia upregulates hypoxia inducible factor (HIF)-3alpha expression in lung epithelial cells: Characterization and comparison with HIF-1alpha. Cell Res 16: 548-558, 2006.

27. Tanaka T, Wiesener M, Bernhardt W, Eckardt KU and Warnecke C: The human HIF (hypoxia-inducible factor)-3alpha gene is a HIF-1 target gene and may modulate hypoxic gene induction. Biochem J 424: 143-151, 2009.

28. Yamashita M, Kumasawa K, Miyake T, Nakamura H and Kimura T: Soluble Flt-1 has cytotoxic effects on BeWo choriocarcinoma cells. Reprod Sci 25: 830-836, 2018.

29. Wei SC, Tsao PN, Weng MT, Cao Z and Wong JM: Flt-1 in colorectal cancer cells is required for the tumor invasive effect of placental growth factor through a p38-MMP9 pathway. J Biomed Sci 20: 39, 2013. 
30. Pencik J, Pham HT, Schmoellerl J, Javaheri T, Schlederer M, Culig Z, Merkel O, Moriggl R, Grebien F and Kenner L: JAK-STAT signaling in cancer: From cytokines to non-coding genome. Cytokine 87: 26-36, 2016.

31. Yue JN, Li WM, Hong WZ, Yang J, Zhu T, Fang Y and Fu WG: MiR-210 inhibits apoptosis of vascular endothelial cells via JAK-STAT in arteriosclerosis obliterans. Eur Rev Med Pharmacol Sci 23 (Suppl 3): S319-S326, 2019.

32. Senft C, Priester M, Polacin M, Schroder K, Seifert V, Kogel D and Weissenberger J: Inhibition of the JAK-2/STAT3 signaling pathway impedes the migratory and invasive potential of human glioblastoma cells. J Neurooncol 101: 393-403, 2011.
33. Jane EP, Premkumar DR and Pollack IF: AG490 influences UCN-01-induced cytotoxicity in glioma cells in a p53-dependent fashion, correlating with effects on BAX cleavage and BAD phosphorylation. Cancer Lett 257: 36-46, 2007.

cc)(ㅇ) This work is licensed under a Creative Commons EY NG ND Attribution-NonCommercial-NoDerivatives 4.0 International (CC BY-NC-ND 4.0) License. 